\title{
Ovine/Caprine Lentivirus Group
}

National Cancer Institute

\section{Source}

National Cancer Institute. Ovine/Caprine Lentivirus Group. NCI Thesaurus. Code

C117715.

A non-taxonomic grouping of lentivuruses that infect wild and domestic sheep and goats. 